\title{
NOTE ON DIRICHLET SERIES. IV. ON THE SINGULARI- TIES OF DIRICHLET SERIES
}

\author{
CHUJI TANAKA
}

Let us put

$$
\begin{aligned}
& F(s)=\sum_{n=1}^{\infty} a_{n} \exp \left(-\lambda_{n} s\right) \\
& \left(s=\sigma+i t, 0 \leqq \lambda_{1}<\lambda_{2}<\cdots<\lambda_{n} \rightarrow+\infty\right) .
\end{aligned}
$$

When we vary coefficients $\left\{a_{n}\right\}$, this change has some influence upon singularities. Concerning this problem, O. Szász $[1$, p. 107] has proved the next theorem, which is a generalization of HurwitzPólya's theorem [2, p. 36]:

O. SzÁsz's Theorem. Let (1) have the finite simple convergenceabscissa $\sigma_{s .}$. If $\lim _{n \rightarrow \infty} \log n / \lambda_{n}=0$, then there exists a sequence $\left\{\epsilon_{n}\right\}$ $\left(\epsilon_{n}= \pm 1\right)$ such that $\sum_{n=1}^{\infty} a_{n} \epsilon_{n} \exp \left(-\lambda_{n} s\right)$ has $\sigma=\sigma$, as the natural boundary.

In this note, we shall prove the following theorem of the same type:

TheOREM. Let (1) have the finite simple convergence-abscissa $\sigma_{3}$. If $\lim _{n \rightarrow \infty} \log n / \lambda_{n}=0$, then there exists a Dirichlet series $\sum_{n=1}^{\infty} b_{n}$ $\exp \left(-\lambda_{n} s\right)$ having $\sigma=\sigma$, as the natural boundary such that

(a) $\left|b_{n}\right|=\left|a_{n}\right|(n=1,2, \cdots)$, and $\lim _{n \rightarrow \infty}\left|\arg \left(a_{n}\right)-\arg \left(b_{n}\right)\right|=0$ or

(b) $\arg \left(b_{n}\right)=\arg \left(a_{n}\right)(n=1,2, \cdots)$, and $\lim _{n \rightarrow \infty}\left|b_{n} / a_{n}\right|=1$.

Proof. On account of $\lim _{n \rightarrow \infty} \log n / \lambda_{n}=0$, and G. Valiron's theorem $[3$, p. 4], we get

$$
\sigma_{e}=\limsup _{n \rightarrow \infty} 1 / \lambda_{n} \cdot \log \left|a_{n}\right| \text {. }
$$

Therefore we can select a subsequence $\left\{\lambda_{n_{i}}\right\}$ such that

(i) $\quad \sigma_{s}=\lim _{n \rightarrow \infty} 1 / \lambda_{n_{i}} \cdot \log \left|a_{n_{i}}\right|$,

(ii) $\quad \liminf _{i \rightarrow \infty}\left(\lambda_{n_{i}+1}-\lambda_{n_{i}}\right)>0, \quad \lim _{i \rightarrow \infty} i / \lambda_{n_{i}}=0$.

Again by G. Valiron's theorem and (3) (i), $G_{1}(s ; \theta, \alpha)$

Received by the editors August 22, 1951 and, in revised form, June 6, 1952. 
$=\sum_{i=1}^{\infty} a_{n_{i}} \exp \left(\alpha \theta / \lambda_{n_{i}}\right) \times \exp \left(-\lambda_{n_{i}} s\right)$ has the simple convergenceabscissa $\sigma_{s}$, where (i) $\theta$ is a real constant, (ii) $\alpha$ is a constant determined later. Hence $G_{2}(s)=\sum_{n} \bigoplus_{\left\{n_{i}\right]} a_{n} \exp \left(-\lambda_{n} s\right)$ is simply convergent at least for $\sigma>\sigma_{s}$. Now let us put

$$
F(s ; \theta, \alpha)=G_{1}(s ; \theta, \alpha)+G_{2}(s),
$$

which is evidently simply convergent at least for $\sigma>\sigma_{\text {s. }}$.

Denote by $E(\theta, \alpha)$ the set of regular points of $F(s ; \theta, \alpha)$ on $\sigma=\sigma_{s}$, which is clearly an open set. Then we can prove that

$$
E\left(\theta_{1}, \alpha\right) \cap E\left(\theta_{2}, \alpha\right)=0
$$

for $\theta_{1} \neq \theta_{2}$.

In fact, if there should exist a point $\xi$ on $\sigma=\sigma_{8}$ such that $\xi \in E\left(\theta_{1}, \alpha\right)$ $\cap E\left(\theta_{2}, \alpha\right) \neq 0$, then $F\left(s ; \theta_{1}, \alpha\right)-F\left(s ; \theta_{2}, \alpha\right)$ would be regular at $s=\xi$. On the other hand, since

$$
\begin{aligned}
F\left(s ; \theta_{1}, \alpha\right)-F(s ; & \left.\theta_{2}, \alpha\right) \\
& =\sum_{i=1}^{\infty} a_{n_{i}}\left\{\exp \left(\alpha \theta_{1} / \lambda_{n_{i}}\right)-\exp \left(\alpha \theta_{2} / \lambda_{n_{i}}\right)\right\} \exp \left(-\lambda_{n_{i}} s\right) \\
& =\sum_{i=1}^{\infty} a_{n_{i}} O\left(1 / \lambda_{n_{i}}\right) \exp \left(-\lambda_{n_{i}} s\right),
\end{aligned}
$$

taking account of (3), G. Valiron's theorem, and Carlson-Landau's theorem [3, pp. 140-141], $F\left(s ; \theta_{1}, \alpha\right)-F\left(s ; \theta_{2}, \alpha\right)$ has the simple convergence-abscissa $\sigma_{s}$, and furthermore it has $\sigma=\sigma_{s}$ as the natural boundary, which contradicts the regularity at $s=\xi$. Hence, (5) holds.

If $E(\theta, \alpha) \neq 0$ should hold for all $\theta, 0 \leqq \theta \leqq \gamma$ ( $\gamma$ a fixed constant), then, by (5), $\{F(s ; \theta, \alpha)\}$ is at most of enumerable power, which contradicts the power of continuum of $\{F(s ; \theta, \alpha)\}$. Hence, for at least one $\theta^{\prime}, E\left(\theta^{\prime}, \alpha\right)=0$ holds. If we put $\alpha=(-1)^{1 / 2}(-1)$, then (a) ((b)) is valid. q.e.d.

\section{REFERENCES}

1. O. Szász, Über Singuläritäten von Potenzreiken und Dirichletschen Reihen am Rande des Konvergenzbereiches, Math. Ann. vol. 85 (1922).

2. E. Landau, Darstellung und Gegründung einiger neurer Ergebnisse der Funktionentheorie, Berlin, 1929.

3. V. Bernstein, Leģons sur les progrès récents de la theorie des séries de Dirichlet, Paris, 1933.

WASEDA UNIVERSITY, TOKYo 